\title{
Pulse dye laser therapy and superficial cryotherapy as a novel combination treatment for hypertrophic scars and keloids
}

\section{lqbal A. Bukhari ${ }^{1,2}$}

${ }^{\text {I} C o n s u l t a n t ~ D e r m a t o l o g i s t, ~ D e r m a t o l o g y ~ D e p a r t m e n t, ~ D r . ~ S u l a i m a n ~ A l H a b i b ~ H o s p i t a l, ~ A l k h o b a r, ~ K i n g d o m ~ o f ~ S a u d i ~ A r a b i a . ~}$ ${ }^{2}$ Professor, Dermatology Department, College of Medicine, Imam Abdulrahman Bin Faisal University (IAU), Dammam, Kingdom of Saudi Arabia

Corresponding author: Prof. Iqbal A. Bukhari, MD, E-mail: dermbukhari@gmail.com,ibukhari@iau.edu.sa

\begin{abstract}
Background: Hypertrophic scars are benign and fibrotic skin lesions caused by defects in the regulation of cellularity during the wound-healingprocess, in which there is higher collagen production and less degradation. Genetic predisposing factors and different skin injuries may play a role in developing these types of lesions. On the other hand, keloids are overgrowths of fibrous tissue outside the original boundaries of trauma, yet these may also occur spontaneously. There are numerous treatment options for both conditions, including silicone gel sheeting, pressure therapy, intralesional triamcinolone acetonide, radiation, laser therapy, cryosurgery, interferon, 5-fluorouracil, and surgical excision as well as a multitude of extracts and topical agents. Objective: The objective was to evaluate the effectiveness of pulse dye laser (PDL) therapy and superficial cryotherapy as a combination treatment for hypertrophic scars and keloids. Method: Four Arabic female patients were seen at the outpatient clinic of the Department of Dermatology at the King Fahd Hospital of the University in Khobar, Saudi Arabia. The patients had keloids and hypertrophic scars. Treatment with cryotherapy every week for three weeks followed by one session of pulsed dye laser was administered rotationally for three to six months until the lesions displayed remarkable physical improvement or complete resolution. Results: All patients experienced significant improvement, showing a reduction in the size, erythema, pliability, and pruritus. None of the hypertrophic scars or keloids deteriorated during the one year of treatment. No complications were noted during the treatment period. Conclusion: Sequential PDL therapy combined with superficial cryotherapy may be an option for treating hypertrophic scars and keloids.
\end{abstract}

Keywords: Hypertrophic scars; Keloids; Pulse dye laser; Cryotherapy

\section{INTRODUCTION}

Hypertrophic scars are benign fibrotic skin lesions caused by defects in regulating fibrous tissue cellularity during wound-healing limited to the area of trauma. While keloids are overgrowths of fibrous tissue outside the original boundaries of trauma and occur secondary to defective wound healing, they may occur spontaneously without previous trauma. Both are abnormal responses to trauma followed by excessive wound tension, inducing fibroblast proliferation and overproduction of dense collagen and glycosaminoglycans [1]. The exact pathogenesis of both conditions has not yet been elucidated. Hypertrophic scars and keloids often have functional, aesthetic, and psychosocial impacts on patients, as highlighted by quality-of-life studies. Numerous variables affect the severity of scarring, including the size and depth of the wound, blood supply to the area, the thickness and color of the skin, and the direction of the scar [2].

Numerous treatment options are available with limited efficacy, including surgical excision, intralesional or topical corticosteroids, other intralesional therapies-

\footnotetext{
How to cite this article: Bukhari IA. Pulse dye laser therapy and superficial cryotherapy as a novel combination treatment for hypertrophic scars and keloids. Our Dermatol Online. 2022;13(1):28-31.

Submission: 24.08.2021; Acceptance: 09.11.2021

DOI: $10.7241 /$ ourd.20221.5
} 
5-fluorouracil (5-FU), bleomycin, and interferontopical imiquimod, compression, cryotherapy, radiation, silicone sheeting, and laser or light-based therapies. Specifically, the biological changes that occur during cryosurgery are tissue injuries in which intracellular ice formation damages mitochondria and endoplasmic reticulum, leading to irreversible cell destruction known as homogenous nucleation, followed by heterogenous nucleation, vascular stasis, and tissue anoxemia, resulting in ischemic necrosis followed by healing and tissue reorganization. In keloids, cryosurgery leads to tenascin expression and IFN-g expression being depleted [3].

Laser and light-based therapies may be classified into three categories: ablative lasers, non-ablative lasers, and non-coherent light sources. Ablative lasers, such as 2,940-nm Er: YAG and 10,600-nm CO lasers, target water in the skin, resulting in local tissue destruction $[4,5]$. Non-ablative lasers, such as 585 or $595-\mathrm{nm}$ pulsed-dye lasers (PDL), target hemoglobin in the blood vessels of the scar [6]. 980-nm diode laser targets hemoglobin and melanin [7]. 1064-nm Nd: YAG and 532-nm Nd: Van lasers primarily damage deep dermal blood vessels [8]. Besides, Nd: YAG may directly suppress fibroblast collagen expression [8,9]. Therefore, it is plausible that non-ablative lasers directly affect the biological function of fibroblasts. Non-coherent light sources include intense pulsed light therapy (IPL), light-emitting diode (LED) phototherapy, also known

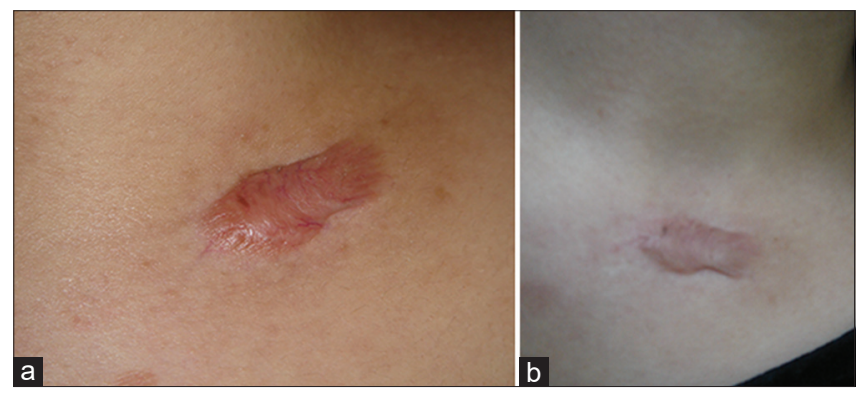

Figure 1: Anterior chest wall keloid (a) before treatment and (b) twelve months after treatment. as low-level light therapy, and photodynamic therapy (PDT). These modalities utilize light energy that may cause fibroblast functional modification [10-13].

This report explored the efficacy of combining the use of PDL and superficial cryotherapy sequentially to treat hypertrophic scar and keloid, which was not reported in previous studies.

\section{METHODS}

Four female patients of Arabic origin were seen at the outpatient clinic of the Department of Dermatology at the King Fahd Hospital of the University in Khobar, Saudi Arabia. Their ages ranged from 24 to 52 years, with a mean of 37 years. Three patients had keloids and one had a hypertrophic scar. The first patient had multiple keloid scars on the back, the second patient had a single keloid on the right shoulder area, the third patient had a single keloid on the upper chest (Fig. la), and the fourth had a hypertrophic scar on the lower abdomen following cesarian section (Fig. 2a), who used topical clobetasol for several months before presentation, then discontinued clobetasol because of its ineffectiveness. The other three patients had received no treatment for their condition. After discussing treatment options with the patients, they agreed to our suggested treatment protocol. Consent was signed by each patient and pre-treatment and posttreatment photographs were taken. Treatment with cryotherapy spray — with a liquid nitrogen canister — was initiated. Each scar was sprayed superficially with two passes on the lesion and 4-mm margins of uninvolved skin. This was performed every week for three weeks, followed by one session of 585-PDL (spot size: $7 \mathrm{~mm}$, fluence: $6-7 \mathrm{~J} / \mathrm{cm}^{2}$, pulse width: $2 \mathrm{~ms}$, repetition rate: $2 \mathrm{~Hz}$ ). Local anesthesia was not necessary in any of the cases. The treatment was repeated rotationally every month for 3-6 months until the lesions displayed physical improvement in the size, erythema, and firmness.

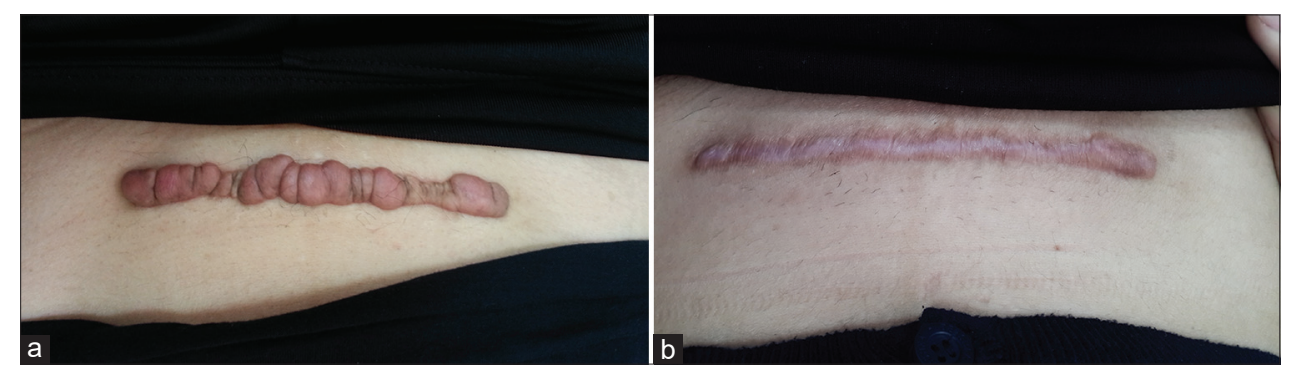

Figure 2: Post-cesarian-section hypertrophic scar (a) before treatment and (b) twelve months after treatment, with the scar completely disappearing with residual post-inflammatory hypopigmentation. 


\section{RESULTS}

All patients experienced significant improvement, showing a reduction in the size, erythema, and firmness (Figs. la and lb). One patient with a hypertrophic scar had complete resolution of the scar with postinflammatory hypopigmentation (Figs. 2a and 2b). None of the hypertrophic scars or keloids deteriorated during the one year of treatment. No complications were noted during the treatment period. There was a subjective decrease in pruritus.

\section{DISCUSSION}

In the 1980s, 585-nm PDL was used to treat scars by coagulation, reducing the redness and thickness of scars [6,14]. Paquet et al. [7] suggested that PDL improves keloids and hypertrophic scars by inducing capillary destruction, which generates hypoxemia and, in turn, alters local collagen production. Dierickx et al. [8] also attributed the therapeutic effect of PDL on hypoxemia, resulting from laser-induced heat and vascular injury. Besides, Kuo et al. [9] found that PDL therapy administered for keloids stimulated the production of matrix metalloproteinase, including collagenase, which contributed to the resolution of scars. To obtain better clinical outcomes, PDL is combined with corticosteroid injections and/or 5 -fluorouracil $[10,11]$. On the contrary, there is a limited penetration depth of the yellow light emitted by PDL because of the optical absorption and scattering in the epidermis and dermis at a depth of about $1-2 \mathrm{~mm}$, causing resistance to further PDL treatment $[12,13]$, which justifies our protocol of combining cryotherapy with PDL in the treatment of hypertrophic scars and keloids to reach deeper tissues without inducing complications. Spraying and contact cryotherapy are older techniques. Intralesional cryotherapy is a relatively novel technique that freezes the scar from the center outwards. A recent review identified intralesional cryotherapy as a safe and effective modality with few adverse effects [15].

Scar outcomes after treatment were sometimes measured with scar-rating systems, such as the Vancouver Scar Scale (VSS) or its modified version. The VSS grades vascularity, thickness, pliability, and pigmentation [16]. However, non-universal use of this assessment scale limits the usefulness in comparing study outcomes. Reductions in the size, erythema, pliability, and symptoms make the clinical assessment of these parameters a more appropriate measurement [17].

In our patients, we found that rotational treatment of hypertrophic scars and keloids with $585 \mathrm{~nm}-\mathrm{PDL}$ and superficial cryotherapy may be a new treatment option with good outcomes.

Finally, it is important to consider the patient's skin type, downtime, and compliance when treating keloids and hypertrophic scars. Future research will enhance our understanding of hypertrophic scars and keloids through newly discovered treatment modalities and, specifically, in light-based technology, leading to superior treatment outcomes [17].

\section{CONCLUSION}

This is the first report showing that treating hypertrophic scars and keloids with 585nm-PDL combined with superficial cryotherapy may be an appropriate, non-ablative option for treating and improving the appearance of hypertrophic scars and keloids. We expect that future reports will support our findings.

\section{Statement of Human and Animal Rights}

All the procedures followed were in accordance with the ethical standards of the responsible committee on human experimentation (institutional and national) and with the 2008 revision of the Declaration of Helsinki of 1975.

\section{Statement of Informed Consent}

Informed consent for participation in this study was obtained from all patients.

\section{REFERENCES}

1. Bimbi C, Brzeziński P. Combined treatment of keloids and scars with Nd: YAG $1064 \mathrm{~nm}$ laser and cryotherapy: Report of clinical cases. Our Dermatol Online. 2020;11:149-53.

2. Talwar A, Puri N. A study on scar revision. Our Dermatol Online. 2016;7:155-9.

3. Thapa DP, Jha AK, Shrestha S, Joshi S. Cryosurgery in a dermatology setup: A hospital-based study. Our Dermatol Online. 2018;9:137-9.

4. Alster TS. Cutaneous resurfacing with $\mathrm{CO} 2$ and erbium: YAG lasers: Preoperative, intraoperative, and postoperative considerations. Plast Reconstr Surg. 1999;103:619-32; discussion 633-4.

5. Wagner JA, Paasch U, Bodendorf MO, Simon JC, Grunewald S. Treatment of keloids and hypertrophic scars with the triple-mode Er: YAG laser: A pilot study. Med Laser. 2011;26:10-5.

6. Alster TS, Handrick C. Laser treatment of hypertrophic scars, keloids, and striae. Seminars in Cutaneous Medicine and Surgery. 2000;19:287-92.

7. Kassab AN, El Kharbotly A. Management of ear lobule 
keloids using 980-nm diode laser. Eur Arch Otorhinolaryngol. 2012;269:419-23.

8. Philipp CM, Scharschmidt D, Berlien HP. Laser treatment of scars and keloids: How we do it. Medical Laser Application. 2008;23:79-86.

9. Abergel RP, Meeker CA, Lam TS, Dwyer RM, Lesavoy MA, Uitto J. Control of connective tissue metabolism by lasers: Recent developments and future prospects. J Am Acad Dermatol. 1984;11:1142-50.

10. Nie Z. Is photodynamic therapy a solution for keloid? Giornale Italiano di Dermatologia e Venereologia. 2011;146:463-72.

11. Kontoes PP, Marayiannis KV, Vlachos SP. The use of intense pulsed light in the treatment of scars. Eur J Plast Surg. 2003;25:374-7.

12. Huang YY, Sharma SK, Carroll J, Hamblin MR. Biphasic dose response in low level light therapy - an update. Dose Response. 2011;9:602-18.

13. Liu A, Moy RL, Ross EV, Hamzavi I, Ozog DM. Pulsed dye laser and pulsed dye laser-mediated photodynamic therapy in the treatment of dermatologic disorders. Dermatol Surg. 2012;38:351-66.
14. Garden JM, Tan OT, Kerschmann R, Boll J, Furumoto H, Anderson RR, et al. Effect of dye laser pulse duration on selective cutaneous vascular injury. J Invest Dermatol. 1986;87:653-7.

15. O'Boyle CP, Shayan-Arani H, Hamada MW. Intralesional cryotherapy for hypertrophic scars and keloids: A review. Scars Burn Heal. 2017;17:3:2059513117702162.

16. Fearmonti R, Bond J, Erdmann D, Levinson H. A review of scar scales and scar measuring devices. Eplasty. 2010;10:e43.

17. Mamalis AD, Lev-Tov H, Nguyen DH, Jagdeo JR. Laser and light-based treatment of Keloids--A review. J Eur Acad Dermatol Venereol. 2014;28:689-99.

Copyright by lqbal A. Bukhari. This is an open-access article distributed under the terms of the Creative Commons Attribution License, which permits unrestricted use, distribution, and reproduction in any medium, provided the original author and source are credited.

Source of Support: Nil, Conflict of Interest: None declared. 